\title{
AN INEQUALITY FOR A STEKLOFF EIGENVALUE BY THE METHOD OF DEFECT ${ }^{1}$
}

\section{J. R. KUTTLER ${ }^{2}$ AND V. G. SIGILLITO}

1. Introduction. In this paper we give a lower bound to the first nonzero eigenvalue $p_{2}$ of the Stekloff problem [8] for plane regions having two axes of symmetry. Such bounds lead to a priori inequalities which are useful in giving error bounds for approximate solutions to the Neumann problem for Poisson's equation (see $[\mathbf{5}, \S 2]$ ).

In [9], Weinstock has given an isoperimetric upper bound for $p_{2}$, but good lower bounds, which are more useful, are more elusive (see [1], [4], [6]).

We first prove a nodal-line theorem of some interest in itself. This nodal-line theorem does not seem to appear anywhere in the literature, although its proof is a straightforward application of familiar methods (see [7]). The results of the nodal-line theorem then permit us to use the method of defect (see, e.g., [2]) to obtain our bound by integrating an easily obtained one-dimensional version of the desired inequality.

2. Preliminaries. Let $B$ be a bounded, connected domain of the $x_{1}, x_{2}$-plane with piecewise smooth boundary $\partial B$. We consider the Stekloff eigenvalue problem

$$
\Delta u=0 \text { in } B, \quad \partial u / \partial n=p u \text { on } \partial B,
$$

where $\Delta$ is the Laplacian and $n$ the unit outer normal on $\partial B$. The problem has a discrete spectrum of eigenvalues $0=p_{1}<p_{2} \leqq p_{3} \leqq \ldots$ with corresponding eigenfunctions $u_{1}=$ constant, $u_{2}, u_{3}, \cdots$. The eigenvalues can be characterized by

$$
p_{n}=\min \frac{\int_{B}|\operatorname{grad} v|^{2} d x}{\oint_{\partial B} v^{2} d s}
$$

where the minimum is taken over all continuous and piecewise continuously differentiable functions $v$ satisfying

$$
\oint_{\partial B} v u_{k} d s=0, \quad k=1,2, \cdots, n-1
$$

Received by the editors October 30, 1967.

${ }^{1}$ Supported in part by Contract No. AS-7-283, Aerospace Research Laboratories, Office of Aerospace Research, United States Air Force.

2 Post-doctoral Fellow at the Applied Physics Laboratory, The John Hopkins University. 
The minimum of (2) is attained when and only when $v$ is an eigenfunction of (1) associated with $p_{n}$.

3. The nodal-line theorem. A curve in $B$ along which an eigenfunction $u_{n}$ vanishes is called a nodal line of $u_{n}$. What we prove is

THeOREM. The nodal lines of $u_{n}$ divide $B$ into no more than $n$ subdomains, and no nodal line is a closed curve.

Proof. We prove the second part of the theorem first. If a nodal line were closed, we would have $u_{n} \equiv 0$ in the interior of the curve since $\Delta u_{n}=0$ in $B$. But then $u_{n}$ would vanish identically in $B$ by the Unique Continuation Theorem for harmonic functions (see, e.g., [3, Chapter $\mathrm{X}, \S 5])$. Thus no nodal line can be a closed curve.

Now suppose that the nodal lines of $u_{n}$ divide $B$ into more than $n$ subdomains. Let $D_{1}, D_{2}, \cdots, D_{n}$ be $n$ of these subdomains. Note that $\partial B \cap \partial D_{i}$ is not empty. Define $w_{i}$ to agree with $u_{n}$ on $D_{i}$ and vanish on $B-D_{i}, i=1,2, \cdots, n$. Notice that $w_{i} \neq 0$ on $\partial B \cap \partial D_{i}$, otherwise $w_{i} \equiv 0$ in $D_{i}$ (since $\Delta w_{i}=0$ in $D_{i}$ ). Thus, we can find a linear combination, say $v=\sum_{i=1}^{n} a_{i} w_{i}$, such that

$$
\oint_{\partial B} v^{2} d s=1
$$

and $v$ satisfies (3). Moreover, the $w_{i}$, hence $v$, are continuous and piecewise continuously differentiable (see [3, Chapter X, §9]). Since $\partial v / \partial n=p_{n} v$ on $\partial B$, it follows from Green's first identity that

$$
\frac{\int_{B}|\operatorname{grad} v|^{2} d x}{\oint_{\partial B} v^{2} d s}=p_{n} .
$$

Thus $v$ minimizes (2) and is therefore an eigenfunction of (1). Hence $v$ is harmonic and vanishes on the nonempty subdomain $B-D_{1}$ $\cup \ldots \cup D_{n}$. Using the Unique Continuation Theorem we arrive at a contradiction. This completes the theorem.

The application we wish to make of this theorem is the following. Since an eigenfunction $u_{2}$ associated with $p_{2}$ satisfies $\oint_{\partial B} u_{2} d s=0$, we see that $u_{2}$ must have a nodal line. By the theorem $u_{2}$ cannot have more than one, hence has exactly one.

4. The method of defect. Suppose now our region $B$ has two distinct axes of symmetry. They may be assumed perpendicular, and, with no loss of generality, we take them to be the $x_{1}$ and $x_{2}$ axes. We will call a function defined on $B$ even-even, odd-odd, even-odd, or odd-even as $u$ is respectively even in both $x_{1}$ and $x_{2}$, odd in both $x_{1}$ 
and $x_{2}$, even in $x_{1}$ and odd in $x_{2}$, or odd in $x_{1}$ and even in $x_{2}$. Every eigenfunction of (1) can be assumed to belong to one of these symmetry classes. From the previous section, $u_{2}$ must be in the even-odd or odd-even symmetry classes. (Otherwise, $u_{2}$ has an even number of nodal lines.) Hence, the nodal line is an axis of symmetry.

The one-dimensional inequality

$$
[u(0)]^{2}+[u(l)]^{2} \leqq \frac{l}{2} \int_{0}^{l}\left[u^{\prime}(y)\right]^{2} d y,
$$

for continuous and piecewise continuously differentiable functions on the interval $(0, l)$ which satisfy $u(0)=-u(l)$, is easily shown by solving the Euler equation.

Let us first consider the case when the eigenfunction $u_{2}$ is odd across the $x_{1}$-axis. Suppose the boundary $\partial B$ can be expressed by $x_{2}$ $= \pm f_{1}\left(x_{1}\right),-a_{1} \leqq x_{1} \leqq a_{1}$. Then, employing (4),

$\oint_{\partial B} u_{2}^{2} d s$

$$
\begin{aligned}
& =2 \int_{-a_{1}}^{a_{1}}\left[u_{2}\left(x_{1}, f_{1}\left(x_{1}\right)\right)\right]^{2}\left(1+\left[f_{1}^{\prime}\left(x_{1}\right)\right]^{2}\right)^{1 / 2} d x_{1} \\
& \leqq \int_{-a_{1}}^{a_{1}}\left(1+\left[f_{1}^{\prime}\left(x_{1}\right)\right]^{2}\right)^{1 / 2}\left[f_{1}\left(x_{1}\right) \int_{-f_{1}\left(x_{1}\right)}^{f_{1}\left(x_{1}\right)}\left[\frac{\partial u_{2}\left(x_{1}, x_{2}\right)}{\partial x_{2}}\right]^{2} d x_{2}\right] d x_{1} \\
& \leqq\left[\max _{-a_{1} \leqq x_{1} \leqq a_{1}} f_{1}\left(x_{1}\right)\left(1+\left[f_{1}^{\prime}\left(x_{1}\right)\right]^{2}\right)^{1 / 2}\right] \int_{-a_{1}}^{f_{1}\left(x_{1}\right)} \int_{-f_{1}\left(x_{1}\right)}\left(\frac{\partial u_{2}}{\partial x_{2}}\right)^{2} d x_{2} d x_{1} \\
& \leqq\left[\max _{-a_{1} \leqq x_{1} \leqq a_{1}} f_{1}\left(x_{1}\right)\left(1+\left[f_{1}^{\prime}\left(x_{1}\right)\right]^{2}\right)^{1 / 2}\right] \int_{B}\left|\operatorname{grad} u_{2}\right|^{2} d x .
\end{aligned}
$$

By similarly treating the case when $u_{2}$ is odd across the $x_{2}$-axis, we have our inequality:

$$
p_{2}^{-1} \leqq \max _{i=1,2}\left[\max _{-a_{i} \leqq x_{i} \leqq a_{i}} f_{i}\left(x_{i}\right)\left(1+\left[f_{i}^{\prime}\left(x_{i}\right)\right]^{2}\right)^{1 / 2}\right],
$$

where $x_{1}= \pm f_{2}\left(x_{2}\right),-a_{2} \leqq x_{2} \leqq a_{2}$, is another representation of $\partial B$.

5. Some examples. We give a few examples of the application of (5) to particular regions. For an upper bound, we use the isoperimetric inequality of Weinstock [9], which says

$$
p_{2} \leqq 2 \pi / L \text {, }
$$

where $L$ is the length of $\partial B$. 
First, consider the rhombus

$$
\left|x_{1} / a_{1}\right|+\left|x_{2} / a_{2}\right| \leqq 1 .
$$

We have $L=4\left(a_{1}^{2}+a_{2}^{2}\right)^{1 / 2}$ and

$$
f_{i}\left(x_{i}\right)=a_{j}\left(1-\left|x_{i} / a_{i}\right|\right), \quad j \neq i .
$$

Thus, from (5) and (6), we have

$$
\min \left(a_{2} / a_{1}, a_{1} / a_{2}\right) \leqq p_{2}\left(a_{1}^{2}+a_{2}^{2}\right)^{1 / 2} \leqq \pi / 2 .
$$

When $a_{1}=a_{2}=S / \sqrt{ } 2$, the rhombus is a square of side $S$, and (7) becomes $1 \leqq p_{2} S \leqq \pi / 2$, whereas the exact value to five places is $p_{2} S$ $=1.3765 \cdots$.

Next, consider the ellipse $\left(x_{1} / a_{1}\right)^{2}+\left(x_{2} / a_{2}\right)^{2} \leqq 1$ for which

$$
f_{i}\left(x_{i}\right)=a_{j}\left(1-\left(x_{i} / a_{i}\right)^{2}\right)^{1 / 2}, \quad j \neq i .
$$

For simplicity, we worsen (6) by combining it with the classical isoperimetric inequality

$$
L^{2} \geqq 4 \pi A,
$$

where $A$, the area of the ellipse, is $\pi a_{1} a_{2}$. Thus, we have

$$
\left[\max \left(a_{1}, a_{2}\right)\right]^{-1} \leqq p_{2} \leqq\left(a_{1} a_{2}\right)^{-1 / 2} \text {. }
$$

For the special case of a disc when $a_{1}=a_{2}=R$, we attain equality on both sides and $p_{2} R=1$.

\section{REFERENCES}

1. J. H. Bramble and L. E. Payne, Bounds in the Neumann problem for second order uniformly elliptic operators, Pacific J. Math. 12 (1962), 823-833.

2. J. Hersch, Sur la fréquence fondamentale d'une membrane vibrante: Evaluations par defaut et principe de maximum, Z. Angew. Math. Phys. 11 (1960), 387-413.

3. O. D. Kellogg, Foundations of potential theory, Dover, New York, 1953.

4. J. R. Kuttler and V. G. Sigillito, Inequalities for Stekloff and membrane eigenvalues, J. Math. Anal. Appl. 23 (1968), 148-160.

5. L. E. Payne, Isoperimetric inequalities and their applications, SIAM Rev. 9 (1967), 453-488.

6. L. E. Payne and H. F. Weinberger, New bounds in harmonic and biharmonic problems, J. Math. Phys. 33 (1955), 291-307.

7. A. Pleijel, Remarks on Courant's nodal line theorem, Comm. Pure Appl. Math. 9 (1956), 543-550.

8. M. W. Stekloff, Sur les problèmes fondamentaux de la physique mathématique, Ann. Sci. Ecole Norm. Sup. 19 (1902), 455-490.

9. R. Weinstock, Inequalities for a classical eigenvalue problem, J. Rational Mech. Anal. 3 (1954), 745-753.

The Johns Hopkins University Applied Physics Laboratory 